\title{
InVESTIgATING THE THERMAl STABILITY OF 1-3 Piezoelectric COMPOSITE TranSDUCERS BY VARYING THE THERMAL CONDUCTIVITY AND GLASS TRANSITION TEMPERATURE OF THE POLYMERIC FILLER MATERIAL.
}

${ }^{1}$ A.C.S. Parr, ${ }^{1}$ R.L. O’Leary, ${ }^{1}$ G. Hayward, ${ }^{1}$ G. Smillie, ${ }^{1}$ G. Benny, ${ }^{2}$ H. Ewing and ${ }^{3}$ A.R Mackintosh. ${ }^{1}$ Centre for Ultrasonic Engineering, Dept. of Electronic and Electrical Engineering, ${ }^{2}$ Metallurgy and Engineering Materials Group, Dept. of Mechanical Engineering, ${ }^{3}$ Dept. of Chemistry, University of Strathclyde, Glasgow G1 1PY, Scotland

\begin{abstract}
The thermal behaviour of a number of 1-3 piezoelectric composite transducers is discussed. In particular, devices manufactured from a polymer filler with a relatively high glass to rubber transition temperature $\left(T_{g}\right)$, and from polymer systems with increased thermal conductivity, are evaluated. The mechanical properties of the various filler materials were obtained via ultrasonic measurements, with the thermal properties extracted using dynamic mechanical thermal analysis (dmta), differential scanning calorimetry (dsc) and laserflash studies. A range of ultrasonic transducers were then constructed and their thermal stability studied using a combination of impedance analysis and laser surface displacement measurement.
\end{abstract}

\section{METHODOLOGY}

It is known that the performance of 1-3 connectivity piezoelectric composite transducers is altered by temperature-induced variations in the device [1]. In this work, six different polymeric materials are investigated. The elastic properties and mechanical attenuation properties of each material were measured using a through transmission ultrasonic technique [2]. Thermal conductivity (k) was determined from thermal diffusivity $(\propto)$ values measured using a laserflash technique. Heat capacity values $(\mathrm{Cp})$ were determined using dsc [3]. The Tg for each material was measured using dmta [4], at the temperature where maximum damping was measured, corresponding to the maximum in $\tan \delta$, where $\tan \delta$ is the ratio of dissipated to stored energy per cycle. Samples of 1-3 piezoelectric composites of, $38 \%$ volume fraction, were manufactured with each polymer as the passive phase. Electrical impedance measurements were conducted in an oven, as a function of polymer temperature and investigations using a laser vibrometer system were also carried out [5].

\section{MATERIALS AND CHARACTERISATION}

A number of epoxy based thermosetting polymers were investigated in this study. Two unfilled polymers, (A) CY1301/HY1300, a hard setting resin, and (B) MY750/HY906/DY062, a high Tg resin, were supplied by Ciba Polymers and Stycast provided two of the polymers containing thermally conductive fillers. These were (C) 2651-40/Cat 9, containing mica and (D) 2850FT/Cat 9, containing aluminium oxide. For all of these commercial materials, polymer samples were prepared in accordance with the manufacturers' instructions [6,7]. MY750/HY906/DY062 was modified within the CUE laboratory to prepare two new polymer systems containing thermally conductive additive where the maximum concentration of thermally conductive additive, to result in a suitable viscosity for filling the diced ceramic bristle block, was used. Sample (E) contained 30vol\% aluminium oxide (Aldrich, 99.7\%) and $(\mathrm{F})$ contained $24 \mathrm{vol} \%$ aluminium nitride (Aldrich, $98+\%)$. Both additives have a particle size of less than 10 micron.

\begin{tabular}{|l|l|l|l|l|l|l|}
\hline Polymer & \multicolumn{1}{|c|}{ A } & \multicolumn{1}{c|}{ B } & \multicolumn{1}{c|}{ C } & \multicolumn{1}{c|}{ D } & \multicolumn{1}{|c|}{ E } & \multicolumn{1}{|c|}{} \\
\hline $\mathbf{V l}(\mathbf{m} / \mathbf{s})$ & 2513 & 2342 & 2925 & 3377 & 2813 & 2636 \\
\hline $\mathbf{V s}(\mathbf{m} / \mathbf{s})$ & 1175 & 1094 & 1494 & 1780 & 1464 & 1345 \\
\hline$\rho\left(\mathbf{k g} / \mathbf{m}^{\mathbf{3}}\right)$ & 1149 & 1150 & 1503 & 2292 & 2007 & 1673 \\
\hline $\boldsymbol{V}$ & 0.360 & 0.360 & 0.323 & 0.308 & 0.314 & 0.324 \\
\hline $\mathbf{E}(\mathbf{G P a})$ & 4.28 & 3.75 & 8.88 & 18.99 & 11.3 & 8.01 \\
\hline $\mathbf{B}(\mathbf{G p a})$ & 5.10 & 4.47 & 8.38 & 16.45 & 10.14 & 7.60 \\
\hline $\mathbf{G ( G p a )}$ & 1.57 & 1.38 & 3.35 & 7.26 & 4.30 & 3.03 \\
\hline $\mathbf{Z}(\mathbf{M r a y l})$ & 2.86 & 2.69 & 4.40 & 7.74 & 5.65 & 4.41 \\
\hline$\alpha_{\mathbf{l}}(\mathbf{d B} / \mathbf{m})$ & 139 & 174 & 176 & 96 & 174 & 174 \\
\hline$\alpha_{\mathbf{s}}(\mathbf{d B} / \mathbf{m})$ & 356 & 243 & 363 & 252 & 400 & 382 \\
\hline
\end{tabular}

Table 1 Acoustic Characterisation $(500 \mathrm{kHz})$ 
The longitudinal, $\mathrm{V}_{\mathrm{l}}$, and shear, $\mathrm{V}_{\mathrm{s}}$, acoustic wave velocities were measured, allowing the calculation of Poisson's Ratio (v), Young's modulus (E), the shear modulus $(\mathrm{G})$ and the specific acoustic impedance $(\mathrm{Z})$ of each material. The attenuation of each wave type, $\alpha_{1}$ and $\alpha_{s}$ was also determined. The salient mechanical and temperature related properties are outlined in Tables 1 and 2 respectively. At a particular temperature, the thermal conductivity of a material will depend upon the thermal diffusivity, specific heat capacity and density of the material at that temperature. In this study, the thermal conductivity at $25^{\circ} \mathrm{C}$ is considered.

\begin{tabular}{|l|l|l|l|l|l|l|}
\hline Polymer & \multicolumn{1}{|c|}{ A } & \multicolumn{1}{c|}{ B } & \multicolumn{1}{c|}{ D } & \multicolumn{1}{|c|}{ E } & F \\
\hline $\begin{array}{l}\mathbf{C p} @ 25^{\circ} \mathbf{C} \\
(\mathbf{k J} / \mathbf{k g K})\end{array}$ & $\mathbf{1 . 3 5}$ & $\mathbf{1 . 2 5}$ & $\mathbf{1 . 1 4}$ & $\mathbf{0 . 9 2}$ & $\mathbf{0 . 6 2}$ & $\mathbf{0 . 8 4}$ \\
\hline $\begin{array}{l}\tan \delta \\
\mathbf{m a x}\left({ }^{\circ} \mathbf{C}\right)\end{array}$ & $\mathbf{6 0}$ & $\mathbf{1 5 1}$ & $\mathbf{8 7}$ & $\mathbf{8 4}$ & $\mathbf{1 5 4}$ & $\mathbf{1 4 5}$ \\
\hline $\begin{array}{l}\text { @ @25 } \mathbf{C}^{\circ} \\
\mathbf{1 0}^{-3}\left(\mathbf{c m}^{2} / \mathbf{s}\right)\end{array}$ & 1.42 & 1.04 & 2.92 & 6.45 & & \\
\hline $\mathbf{K}(\mathbf{W} / \mathbf{m K})$ & $0.22^{6}$ & $0.15^{6}$ & $0.5^{7}$ & $1.36^{7}$ & $\mathbf{0 . 3 8}$ & $\mathbf{0 . 4 1}$ \\
\hline
\end{tabular}

Table 2 Thermal Behaviour where Italic is Calculated value and Bold is Measured Experimental value

It is generally recognised that the thermal conductivity of a polymer is increased as the concentration of the thermally conductive additive is increased [8] and the thermal conductivity measured for a polymer/thermally conductive particle combination is much lower than reported for the thermal conductivity of the additive prior to mixing [8]. In this study it was found that very similar values were calculated at $25^{\circ} \mathrm{C}$ for the thermal conductivity of (E) and (F). Higher values are reported for samples (C) and (D) respectively, indicating higher additive concentrations. This correlates with the data from the acoustic characterisation measurements.

\section{TRANSDUCER EVALUATION}

Six piezoelectric composite devices of $38 \%$ ceramic volume fraction were constructed, employing each of the different polymers, as detailed in Table 3. Each device had three K-type thermocouples embedded in the polymer kerf, at half the pillar height.

To investigate the effect of temperature on electrical impedance, the impedance profile of each device was recorded, using a HP4194A impedance analyser, as a function of temperature. The frequency and magnitude of the thickness mode impedance minimum (electrical resonance $-\mathrm{f}_{\mathrm{e}}$ ) and impedance maximum (mechanical resonance - $f_{m}$ ) were examined in detail. Pillar displacement was measured on two devices using the laser vibrometer [5]. By controlling the input voltage into the system at a particular frequency, it was possible to induce temperature increases in the transducer.

\begin{tabular}{|l|c|c|c|c|c|c|}
\hline Device & A & B & C & D & E & F \\
\hline Polymer & A & B & C & D & E & F \\
\hline Ceramic (PZT) & $5 \mathrm{~A}$ & $5 \mathrm{~A}$ & $5 \mathrm{~A}$ & $5 \mathrm{~A}$ & $5 \mathrm{~A}$ & $5 \mathrm{~A}$ \\
\hline Kerf (mm) & 0.63 & 0.63 & 0.63 & 0.61 & 0.61 & 0.66 \\
\hline Pitch (mm) & 1.73 & 1.73 & 1.68 & 1.71 & 1.68 & 1.76 \\
\hline Thickness (mm) & 4.87 & 5.07 & 3.57 & 4.29 & 4.89 & 4.92 \\
\hline fe (kHz) & 299 & 290 & 437 & 388 & 313 & 307 \\
\hline fm (kHz) & 364 & 346 & 513 & 435 & 360 & 360 \\
\hline
\end{tabular}

Table 3 Constructional Parameters of the Six Piezoelectric Composite Devices

(a) Transducer Devices Containing Unfilled Polymers

For device A, clear changes in the electrical impedance were observed at approximately $30^{\circ} \mathrm{C}$, around half of the measured Tg. As the polymer starts to soften, the thickness resonant frequency decreases until the onset of decoupling, when the ceramic phase starts to vibrate independently of the filler. This was observed to occur at approximately $90^{\circ} \mathrm{C}-100^{\circ} \mathrm{C}$ and is illustrated very clearly in Figure 1, which depicts the magnitude and phase of the surface displacement at $28^{\circ} \mathrm{C}$ and $100^{\circ} \mathrm{C}$, over a central square area encompassing 9 pillars.

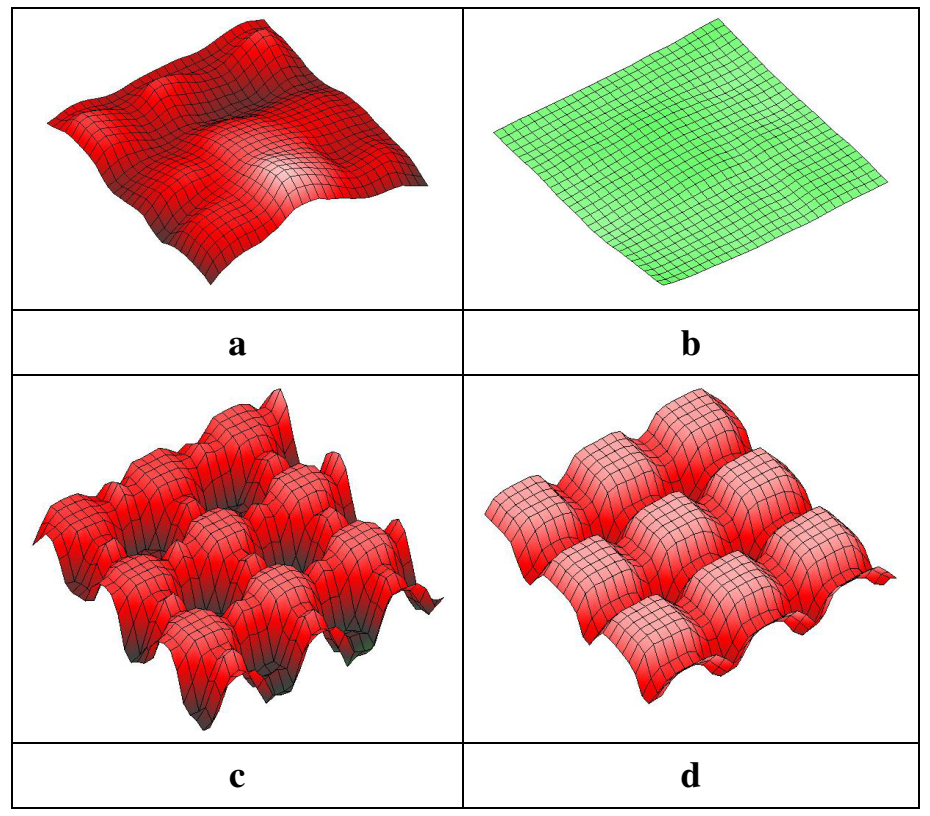

Figure 1 Decoupling effects at high temperature in Device A (a) Amplitude $28^{\circ} \mathrm{C} / 280 \mathrm{kHz}$, (b) Phase $28^{\circ} \mathrm{C} / 280 \mathrm{kHz}$ ), (c) Amplitude $100^{\circ} \mathrm{C} / 280 \mathrm{kHz}$ and (d) Phase $100^{\circ} \mathrm{C} / 280 \mathrm{kHz}$ ) 
In Figures $1 \mathrm{a}$ and $1 \mathrm{~b}$, it is apparent that a relatively uniform displacement is being recorded. However, at $100^{\circ} \mathrm{C}$ the ceramic displacement is dominant and moreover, both materials are vibrating with essentially out of phase motion.

The impedance data generated from oven testing of device B suggested that use of a high glass transition temperature polymer filler material had enhanced the temperature stability of the piezoelectric composite device. However, although the device appeared stable until $140^{\circ} \mathrm{C}$ and maximum loss was recorded at $170^{\circ} \mathrm{C}$, detailed examination indicated that subtle changes were occurring at temperatures as low as $30^{\circ} \mathrm{C}$.

Laser testing confirmed that performance changes in both composites can occur at temperatures not far above ambient. Some results are summarised in Figures 2 and 3 , where the changes that occurred in the frequency of the electrical resonance as a result of external temperature changes during oven testing, are compared with the changes that were observed in the frequency of maximum surface displacement, at different induced temperatures during laser testing, for piezoelectric composite A and piezoelectric composite B respectively.

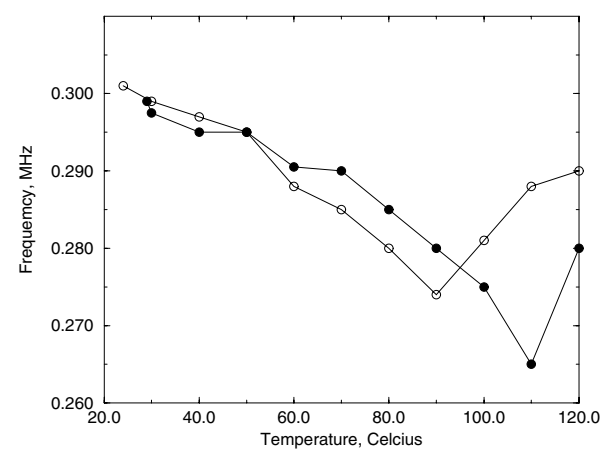

Figure 2 Effect of Device Temperature on device A where (O) denotes frequency of $f_{e}$ in oven tests and ( $\bullet$ ) denotes frequency of maximum relative surface displacement in laser tests

Somewhat surprisingly, the results of the oven and laser tests compare well, although the onset of decoupling appears higher from the laser data in Fig 2. In both cases, the frequency of the impedance minimum at the thickness mode resonance is observed to decrease steadily with temperature. As stated previously, this is indicative of polymer softening and although the higher $\mathrm{Tg}$ device demonstrates a much wider range before the onset of pillar decoupling, softening is occurring at a much earlier stage.

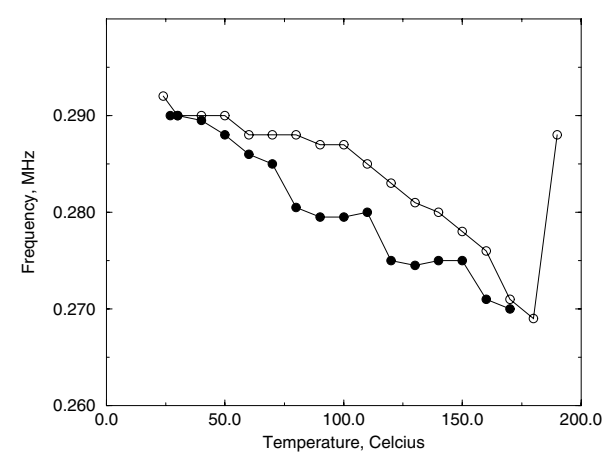

Figure 3 Effect of Device Temperature on device $B$ where $(O)$ denotes frequency of $f_{e}$ in oven tests and ( $(\bullet)$ denotes frequency of maximum relative surface displacement in laser tests

\section{(b) Transducer Devices Containing Filled Polymers}

Oven data of electrical impedance for piezoelectric composite (E) are presented in Figure 4. This device demonstrated maximum loss at $180^{\circ} \mathrm{C}$ which was slightly higher than recorded for the unloaded high $\mathrm{Tg}$ system (B). However, close study reveals that changes are occurring once again at much lower temperatures. This particular design has an additional mode in the vicinity of the main thickness resonance. It is an overtone of the transducer width resonance and due to the relatively low loss in the high $\mathrm{Tg}$ polymer, is interacting with the main mode. The coupling of the two modes is observed to vary as a function of temperature, particularly between $90^{\circ} \mathrm{C}$ and $150^{\circ} \mathrm{C}$. The additional mode, was not so problematic in device $\mathrm{B}$, as the shear velocity is much higher in the loaded polymer. This illustrates a fundamental design requirement for unimodal operation at the desired thickness mode resonance.

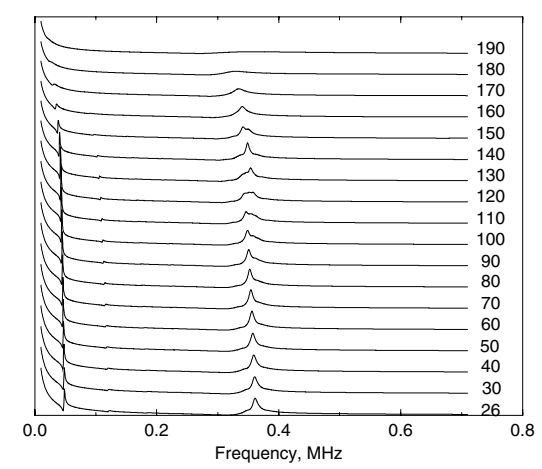

Figure 4 Effect of Temperatur e $\left({ }^{\circ} \mathrm{C}\right)$ on Device $E$ 
Results of electrical impedance testing of piezoelectric composite $(\mathrm{F})$, are shown in Figure 5. This device demonstrated maximum loss at $190^{\circ} \mathrm{C}$, which was slightly higher than recorded for device (E). Only small changes in the frequency and magnitude of the electrical and mechanical resonances were recorded at temperatures of $30-50^{\circ} \mathrm{C}$. Interference from overtones of the transducer width mode was not a problem because of the lower velocity values in polymer $(\mathrm{F})$.

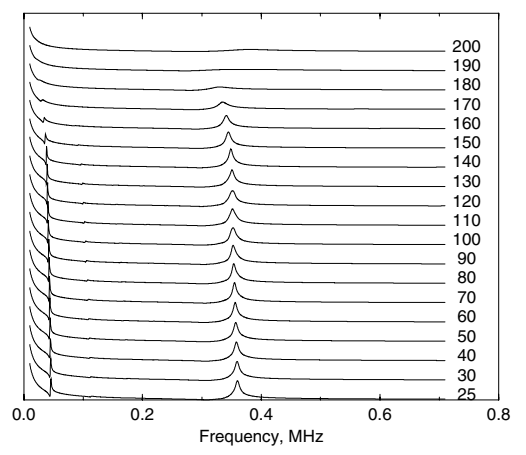

\section{Figure 5 Effect of Temperature $\left({ }^{\circ} \mathrm{C}\right)$ on Device $\mathrm{F}$}

For device C, manufactured from Stycast 2651-40, the $\mathrm{Tg}$ was recorded at $87^{\circ} \mathrm{C}$ and a maximum loss was recorded at $130^{\circ} \mathrm{C}$. For piezoelectric composite (D), manufactured from Stycast 2850-FT, the $\mathrm{Tg}$ was measured at $84^{\circ} \mathrm{C}$ and a maximum loss was recorded at $170^{\circ} \mathrm{C}$ but an overtone from the transducer width mode, was observed as an additional mode in the vicinity of the main thickness resonance. This polymer has relatively high longitudinal and shear velocity values, low Poisson's ratio and very low longitudinal and shear attenuation values which explains why such effects were observed

\section{CONCLUSIONS}

Images obtained in laser displacement studies illustrated the change in surface displacement profile that occurs when decoupling has occurred in a 1-3 piezoelectric composite as a result of induced temperature increases. The ceramic and polymer phases vibrate essentially out of phase. Such behaviour will be typical of that occurring in a device manufactured from a soft polymer. Increasing the $\mathrm{Tg}$ of the polymer filler material, from $60^{\circ} \mathrm{C}$ to $151^{\circ} \mathrm{C}$, improved the thermal stability by extending the operational temperature range of the device but, subtle changes due to softening, observed at temperatures above $30^{\circ} \mathrm{C}$, were still detected in both oven and laser studies. The low thermal conductivity of the polymeric passive phase was considered significant.
The best overall performance was found for the 1-3 piezoelectric composite transducer manufactured from the high Tg polymer modified with aluminium nitride The thermal conductivity, acoustic velocities and density for the passive phase polymer in this device are higher than measured for the high $\mathrm{Tg}$ polymer with no additive but, in addition, advantageously, the shear attenuation has been increased. It is anticipated that devices manufactured from this material will demonstrate stable thickness mode characteristics in water in planned surface displacement studies. Future work involves detailed study of the thermally conductive additive. To optimise acoustic and thermal properties of the polymeric materials, the particle shape and size will be varied and different combinations will be examined. A resin with a higher $\mathrm{Tg}$ will also be investigated. A polymer, prepared from a benzoxazime molecule has been identified with low viscosity and $\mathrm{Tg}$ above $300^{\circ} \mathrm{C}$. Polymers with a particular combination of characteristics, such as high $\mathrm{Tg}$, high attenuation and high thermal conductivity are required to develop devices with superior properties.

Acknowledgements - The authors would like to express their gratitude to the UK Engineering and Physical Sciences Research Council, from whom funding for this study was received.

\section{REFERENCES}

1. A.C.S. Parr et al., "Experimental and Theoretical Evaluation of the Thermal Behaviour of 1-3 Piezoelectric Composite Transducers", Proceedings of IEEE Ultrasonics Symposium, 2000, pp. 1041-1044.

2. H.J. McSkimmin, "Ultrasonic Methods for Measuring the Mechanical Properties of Solids and Liquids", Physical Acoustics, Vol. 1 Part A. Ed. W.P. Mason.

3. J. O'Neill, "Measurement of Specific Heat Functions by Differential Scanning Calorimetry", Analytical Chemistry, Vol. 38, pp 1331-1336, 1966.

4. J.M.G. Cowie, Polymers: Chemistry and Physics of Modern Materials, Intertext books, London, 1973.

5. P. Reynolds, "Analysis and Design of Piezocomposite Ultrasonic Transducers using Finite Element Technique and Surface Displacement Profiles", Ph.D. Thesis, University of Strathclyde, Glasgow, Scotland, 1998.

6. Cold Curing Araldite Resin Systems, Ciba Polymers, Duxford, Cambridge, CB2 4QA, U.K.

7. Stycast Product Literature, Emerson and Cuming Speciality Polymers, London, NW10 7PN, U.K.

8. J.W. Bae et al, "The properties of AlN-filled epoxy molding compounds by the effects of filler size distribution", Journal of Materials Science, 35, 5907-5913, 2000. 